\title{
Development of a new approach for standardization of Centaurium Erythraea Rafn. herb by HPLC method
}

Svitlana M. Gubar, Anna S. Materiienko, Nataliia M. Smielova, Liana G. Budanova, Victoriya A. Georgiyants National University of Pharmacy, Kharkiv, Ukraine

INTRODUCTION: Development a new, fully validated HPLC method for the quantitative analysis of secoiridoid glycosides by an active marker swertiamarin in the Centaurium erythraea herb. The article describes a new approach to the standardization of the Centaurium erythraea Rafn. herb and more specifically the development of a new validated HPLC method for the quantitative determination of secoiridoid glycosides the active marker swertiamarin.

METHODS: Swertiamarin quantitative determination has been estimated in an isocratic mode, on a Symmetry C18 column, using water and acetonitrile as solvents for the mobile phase.

RESULTS: Validation characteristics of the developed method have shown that it is linear in the whole range of concentrations from $0.01 \mathrm{mg} / \mathrm{ml}$ to $0.05 \mathrm{mg} / \mathrm{ml}$ of swertiamarin. All validation characteristics meet the established acceptance criteria.

DISCUSSION AND CONCLUSION: This method can be used in the standardization of raw materials, as well as in the analysis of medicinal products and dietary supplements, which include the Centaurium erythraea herb. The established chromatographic method has been successfully applied for the analysis of raw materials of the Centaurium erythraea Rafn. herb with the quantitative content determination of swertiamarin in the analyzed samples.

Keywords: common centaury herb, development, HPLC, swertiamarin, validation

\section{INTRODUCTION}

Centaurium erythraea Rafn. is a valuable source of various biologically active compounds (BAC), among which are bitters $^{1}$. Due to the presence of this particular group of BAC, the plant is widely used to treat diseases of the gastrointestinal tract and included in the composition of drugs and dietary supplements ${ }^{2}$. The main representatives of secoiridoid glycosides, which determine the pharmacological action of this raw material, are swertiamarin, sweroside and gentiopicroside (Fig. 1).

Bitters improve intestinal motility, increase the reduced secretory function of the stomach and are used in the treatment of hypoacidic and chronic atrophic gastritis, as well. In addition, secoiridoid glycosides show antiinflammatory and antibacterial activity

The results of studies of the biological action of one of the representatives of secoiridoid glycosides of centaury, namely, swertiamarin, are widely described in the literature. It has antihyperlipidemic ${ }^{4}$, hypoglycemic ${ }^{5}$, insulinotropic $^{6,7}$ and antinociceptive ${ }^{8}$ actions. In addition, swertiamarin exhibits an anticholinergic effect ${ }^{9}$, depressant effect and inhibition of human DNA lipase and a CNS depressant effect ${ }^{10}$ as well as inhibits the growth of Bacillus cereus, Bacillus subtilis, Citrobacter freundii, Escherichia coli, Proteus mirabilis and Serratia marcescens ${ }^{2}$.

It is significant to mention that there are only a few publications regarding the description of the methods of Centaurium erythraea analysis. Thus, L. Kaluzova et al. ${ }^{11}$ describes in the studies the analysis of gentiopicroside by HPLC in the Centaurium erythraea herb. P. Valentao et al. ${ }^{12}$ has analyzed xanthones (validation is not described). Z. Glatz et $\mathrm{al}^{13}$ has described the method for determining gentiopicroside in extracts of the Centaurium erythraea using micellar electrokinetic capillary chromatography, and Boryana NikolovaDamyanova and Nedjalka Handjieva ${ }^{14}$ have carried out a quantitative determination of swertiamarin and gentiopicroside using the densitometric method.

In the European Pharmacopoeia, there are no methods for the quantitative analysis of secoiridoid glycosides in this raw material ${ }^{15}$. In the article on the Centaury for the quantitative evaluation of raw materials it is suggested to use the pharmacognosy method "Bitterness value", an organoleptic method of determination, which is based on the individual sensitivity of taste receptors and the subjective assessment of each expert.

The rate of bitterness represents the reciprocal dilution of the mixture, liquid or extract, which still has a bitter taste. This indicator is determined by comparison with quinine hydrochloride, which rate of bitterness is 200,000 (Eur.Ph. 2.8.15). This method is characterized by great subjectivity and high inaccuracy of determination that does not allow recommending it for the analysis of raw materials in modern conditions.

Also in the literature described and studied that representatives of secoiridoid glycosides have different values of bitterness ${ }^{15}$. Proved that swertiamarin is not the most bitter of them, and such a method, as an bitterness value, does not allow to objectively evaluate the content of swertiamarin in the raw material.

The European Pharmacopoeia ${ }^{16}$ suggests the use of swertiamarin as a marker compound during the test

"Identification" by the TLC method. Based on this, as well as on the results of the study of its biological activity, 
we suggested choosing swertiamarin as an active marker in the development of methods for standardization of the Centaurium erythraea herb.

The literature describes approaches to the quantitative determination of swertiamarin in other types of raw materials, such as estimation of swertiamarin in Enicostemma littorale ${ }^{17}$, analysis of swertiamarin from different Swertia species $^{18}$. When developing the methodology, all the described approaches to the analysis of swertiamarin were studied and a new selective, sensitive and accurate HPLC method for the quantitative determination of swertiamarin in Centaurium Erythraea Rafn. herb was developed. The most optimal conditions for the quantitative analysis of swertiamarin in common centaury herb by the HPLC method are proposed: isocratic elution mode and more acceptable chromatographic time, based on the characteristics of this raw material in order to ensure maximum specificity (exclusion of influence on the analysis of ballast substances and other BAS groups of this raw material).

During the course of the experiment, a new, fully validated method was developed for the quantitative analysis of secoiridoid glycosides by the active marker - swertiamarin in Centaurium erythraea herb with HPLC method. This method can be used in the standardization of raw materials, as well as in the analysis of medicinal products and dietary supplements, which include the Centaurium erythraea herb.

\section{EXPERIMENTAL}

HPLC

A quantitative analysis of swertiamarin in the Centaurium erythraea herb has been carried out on a ProStar liquid chromatograph, equipped with an autosampler 410 and two detectors - spectrophotometric PDA 325 and photodiode array detector PDA 330, made by «Varian Chromatography System» (the USA). A Symmetry C18 column $(150 \times 4.6 \mathrm{~mm}$, particle size $3.5 \mu \mathrm{m})$ with a precolumn has also been used. A mixture of water and acetonitrile at the ratio of 91: 9 was used as the mobile phase. The separation has been carried out in an isocratic mode. The flow rate of the mobile phase was $1 \mathrm{ml} / \mathrm{min}$, the injection volume was $20 \mu \mathrm{l}$, the detection has been carried out at a wavelength of $238 \mathrm{~nm}$ and a column temperature was $40^{\circ} \mathrm{C}$.

The sampling has been carried out on an analytical balance "Ohaus Adventurer" brand AR2140 (the USA) by standard procedure for raw materials, according to European Pharmacopoeia (Eur.Ph. 2.9.12). In the process of sample preparation, the following things have also been used: a 355 sieve (SL-200), a medical laboratory centrifuge with a rotor RU-180 OPN-12 "OAO TNK DASTAN"

Raw materials and reagents

To conduct the research, 20 series of Centaurium erythraea herb have been collected in various regions of Ukraine during the flowering period. Macroscopic and microscopic identification of raw materials has been carried out at the Department of Botany of the National University of Pharmacy. The plant species have been deposited in the herbarium section of the Botany Department in National University of Pharmacy, Ukraine (code - BDC 12703).

The following reagents were used: acetonitrile (Sigma Aldrich, gradient grade, for HPLC), methanol (Sigma Aldrich, gradient grade, for HPLC), and water for chromatography (Millipore). Standard sample of swertiamarin (purity - 99.5\%), series OS10475 ("Carbosynth", UK).

Solutions for the analysis have been prepared according to the following methods:

Test solution: $0.500 \mathrm{~g}$ (accurately weighed) of the powdered raw material (355 $\mu \mathrm{m}$, Eur.Ph. 2.9.12) is supplemented with $20 \mathrm{ml}$ of methanol, shaken for 15 minutes, centrifuged and the supernatant is removed into a $50 \mathrm{ml}$ volumetric flask. The extraction is repeated with a further $20 \mathrm{ml}$ of methanol collecting the supernatant as before. The volume of the solution is made up to the mark with methanol and mixed. $10 \mathrm{ml}$ of the obtained solution is diluted to $50 \mathrm{ml}$ with water. The solution is filtered through a $0.45 \mu \mathrm{m}$ membrane filter.

Reference solution: $0.010 \mathrm{~g}$ of swertiamarin (accurately weighed) is placed into a $100 \mathrm{ml}$ volumetric flask, dissolved in $50 \mathrm{ml}$ of methanol, and then the volume is made up to the mark with the same solvent and mixed. 10 $\mathrm{ml}$ of the obtained solution is diluted to $50 \mathrm{ml}$ with water, mixed and filtered through a $0.45 \mu \mathrm{m}$ membrane filter.

\section{Validation}

Validation of the developed method was carried out in accordance with the recommendations of $\mathrm{ICH}^{19}$, the requirements of the article 2.2.N.2 of $\mathrm{SPhU}^{20}$ and according to the standard procedure of quantitative methods validation using an external standard by studying its linearity, as well as accuracy, robustness and precision. To study the specificity, the following solutions were prepared: a blank solution, a reference solution (a solution of a standard sample of swertiamarin) and a test solution.

To confirm the linearity of the method, 5 model solutions were prepared, the concentration of which varies uniformly within the application range to the extent of 50-250\% (step - 50\%).

To determine the accuracy and precision within the range of use of the analytical method, five test solutions were prepared, in compliance with all the stages of the analytical procedure. The concentration of swertiamarin in the prepared solutions ranged from $0.01 \mathrm{mg} / \mathrm{ml}$ to $0.05 \mathrm{mg} / \mathrm{ml}$.

In order to determine the intra-laboratorial precision, one sample were examined six times by two analysts on different days during one working week using various measuring glassware. 


\section{RESULTS AND DISCUSSION}

Analysis of medicinal plant raw materials

HPLC method has been developed for analyzing the quality control of the medicinal plant raw material Centaurium erythraea herb. Swertiamarin has been chosen as the active marker, with the help of which standardization of the raw material is suggested to use, as it was previously established that the centaury herb contained the highest amount of swertiamarin and other secoiridoid glycosides, such as sweroside and gentiopicroside, in a smaller amount ${ }^{21}$.

The HPLC method for analyzing secoiridoid glycosides in the centaury herb has been developed on the basis of the State Research Laboratory for Quality Control of Medicines of the NUPh.

The results of quantitative determination of the swertiamarin content in the analyzed samples of the medicinal plant raw materials are shown in Table 1.

\section{Method validation}

When choosing the criterion for rationing the quantitative content of swertiamarin in the centaury herb, we used the results of the analysis of the raw materials in all indicators applicable to medicinal plant raw materials. It was found that the raw materials, in which the content of swertiamarin was less than $3 \%$, did not meet the requirements of the Pharmacopoeia for such parameters as "Foreign matter" and "Total ash". On this basis, the quantitative content of swertiamarin in the centaury herb was not less than $3 \%$ in terms of dried raw materials. The results obtained during the analysis showed that 15 series of raw materials met these requirements. The total uncertainty of the developed method was calculated, which in this case is related to the limits of the analyte content in the medicinal plant raw materials. For the centaury herb, the established content of swertiamarin is normalized at a level of at least 3\%. In accordance with the requirements of SPhU 2.0 for the quantitative determination (one-sided rationing "no more"), the maximum permissible total uncertainty of the analysis method is $\max \triangle_{A S}<6.4 \%{ }^{17}$.

The criterion of insignificance compared with the maximum permissible uncertainty of the results $\left(\Delta_{A S}\right.$, insig): $\Delta_{A S}$, insig $\leq \max \triangle_{A S}, \% * 0,32=6,4 \% * 0,32=2,048 \%$.

The calculation of the uncertainty of the final analytical operation $\Delta_{F A O}$ is carried out for the test solution and the reference solution. When calculating the intervals, the one-sided coefficient of Student is used for a probability of $95 \%$ and the corresponding number of freedom degrees. Confidence intervals for the reference solution and the testing solution are calculated for an average of five results.

According to the requirements of suitability of the chromatographic system in the determination procedure, the relative standard deviation for five parallel determinations should be no more than $2.0 \%$.

When $\mathrm{n}=5$, $\mathrm{t}(95 \%, \mathrm{n}-1)=2,1318$ :

$$
\Delta_{F A O}^{c m}=\frac{1}{\sqrt{5}} * 2,1318 * 2,0 \%=1,907 \% \Delta_{F A O}^{\operatorname{smp}}=\frac{1}{\sqrt{5}} * 2,1318 * 2,0 \%=1,907 \%
$$

The total uncertainty of the final analytical operation: $\Delta_{F A O}=\sqrt{\left(\Delta_{F A O}^{s m p}\right)^{2}+\left(\Delta_{F A O}^{c m}\right)^{2}}=2,70 \%$

Complete uncertainty of the analysis techniques $\Delta_{A S} \%$ :

$$
\Delta_{A S}=\sqrt{\left(\Delta_{s p}\right)^{2}+\left(\Delta_{F A O}\right)^{2}}=3,39 \%
$$

Thus, the calculated total uncertainty of the analysis $\Delta_{\mathrm{AS}} \%$, which is less than $\max \Delta_{A S}\left(3,39 \%<\max \Delta_{A S}=6,4\right.$ $\%)$, which meets the requirements for this parameter.

Specificity

Under the conditions of the developed method, the determination of the active substance of swertiamarin is not interfered either with the solvent or mobile phase, or other co-eluting impurities from the raw material at a detection wavelength of $238 \mathrm{~nm}$, which indicates the specificity of the developed method.

Chromatograms of the blank solution, the test solution and the reference solution are shown in Fig. 2 in order to confirm the specificity of the method.

\section{Linearity}

The method of quantification must be linear within the application range, and must cover the possible values of the active substance concentrations. According to the requirements of the State Pharmacopoeia of Ukraine, the application range of the method of quantitative determination of swertiamarin in the medicinal plant raw materials must be from 50 to $250 \%$.

Chromatograms of the studying solutions are shown in Fig. 3

The linearity curve is presented in Fig. 4.

The linearity parameters, which are presented in Table 2 , indicate the linearity of the method within the test range.

The obtained results confirm that the developed method for the quantitative determination of swertiamarin by HPLC in the concentration range from $0.01 \mathrm{mg} / \mathrm{ml}$ to $0.05 \mathrm{mg} / \mathrm{ml}$ is linear.

Accuracy, precision and intermediate precision 
Accuracy is characterized by two criteria:

- criterion of statistical insignificance: $\delta \%=\mid \overline{Z-100 \leq \frac{\Delta z}{\sqrt{5}}}$

$\delta \%$ - criterion of practical insignificance - in case if the above ratio is not satisfied, we must use the criterion of insignificance of this systematic error compared with the maximum allowable uncertainty of the analysis:

$|\bar{Z}-100| \leq \Delta_{A S, i n \sin g}=2,048 \%$

The fulfillments of the criteria of accuracy, precision and intermediate precision for determining swertiamarin in the common centaury herb by HPLC are given in Table. 3

The method of determining swertiamarin in the centaury herb satisfies the criteria for acceptability of the validity indicators "Accuracy", "Precision" and "Intermediate precision".

Stability

The study of the stability of the reference solution has been carried out immediately after the preparation, 12 hours and 24 hours later. The results are presented in Table 4.

Differences between the obtained values of the swertiamarin content must not exceed the criterion of

insignificance in comparison with the maximum permissible uncertainty of the analysis results $\left(\triangle_{A S}\right.$, insig $)$, that is

$2.048 \%$. According to the results of the determination, it has been found out that for optimal chromatographic

conditions it is necessary to use a freshly prepared comparison solution within 12 hours which means in term of one working day.

\section{CONCLUSIONS}

This article presents a new method for the quantitative determination of swertiamarin as an active marker for the standardization of raw materials i.e. the Centaurium erythraea herb. The developed method has been fully validated and can be used to control the quality of both the raw material (the Centaurium erythraeaherb), and in the analysis of medicinal products and dietary additives that include this plant.

\section{REFERENCES}

1. $\quad$ European Medicines Agency (EMA), Assessment report on Centaurium erythraea Rafn. s.l. including C. majus (H. et L.) Zeltner and C. suffruticosum (Griseb.) Ronn., herba, for the development of a community herbal monograph, Doc. Ref.: EMA/HMPC/105535/2008, 2009.

2. Kumarasamy, Y., Nahar, L., Cox, P. J., Jaspars, M., \& Sarker, S. D. Bioactivity of secoiridoid glycosides from Centaurium erythraea. Phytomedicine 2003;10:344.

3. Tuluce, Y., Ozkol, H., Koyuncu, I., \& Ine, H. Gastroprotective effect of small centaury (Centaurium erythraea L) on aspirin-induced gastric damage in rats. Toxicol Ind Health 2011;27:760-68.

$4 . \quad$ Vaidya H., Rajani, M., Sudarsanam, V., Padh, H., \& Goyal, R. Swertiamarin: A lead from Enicostemma littorale Blume. for anti-hyperlipidaemic effect. Eur J Pharmacol 2009;617:108-12.

5. Patel M. B., Mishra S. H. Hypoglycemic activity of C-glycosyl flavonoid from Enicostemma hyssopifolium. Pharmaceutical biology 2011;49:383-91.

6. Vaidya H. Vaidya, H., Prajapati, A., Rajani, M., Sudarsanam, V., Padh, H., \& Goyal, R. K. Beneficial Effects of Swertiamarin on Dyslipidaemia in Streptozotocin-induced Type 2 Diabetic Rats. Phytotherapy Research 2012;26:1259-61.

7. Sonawane R. D. Sonawane, R. D., Vishwakarma, S. L., Lakshmi, S., Rajani, M., Padh, H., \& Goyal, R. K. (2010) Amelioration of STZ-induced type 1 diabetic nephropathy by aqueous extract of Enicostemma littorale Blume and swertiamarin in rats. Molecular and cellular biochemistry 2010;340:1-6. 8. Jaishree V., Badami S. Antioxidant and hepatoprotective effect of swertiamarin from Enicostemma axillare against D-galactosamine induced acute liver damage in rats. J Ethnopharmacol 2010;130:103-6.

9. Yamahara J, Kobayashi M, Matsuda H, Akoi S. Anti-cholinergic action of Swertia japonica and an active constituent. J Ethnopharmcol 1991; 3:31-6

10. Bhattacharya SK, Reddy PK, Ghosal S, Singh AK, Sharma PV. Chemical constituents of Gentinaceae XIX: CNS-depressant effects of swertiamarin. J Pharm Sci 1976;65:1547-49.

11. L. Kaluzova, Z. Glatz, J. Pospisilova, P. Musil, J. Unar, Determination of gentiopicroside in Centaurium erythraea by high-performance liquid chromatography. Cesk. Farm. 1995;44:203-5.

12. P. Valentao, P.B. Andrade, E. Silva, A. Vicente, H. Santos, M.L. Bastos, R.M. Seabra,

Methoxylated xanthones in the quality control of small centaury (Centaurium erythraea) flowering tops. J. Agric. Food Chem. 2002;50:460-63.

13. Glatz, Z., Pospísilová, J., \& Musil, P. Determination of gentiopicroside in extracts of Centaurium erythreae and Gentiana lutea by micellar electrokinetic capillary chromatography. Journal of Liquid Chromatography \& Related Technologies 2000;23:1831-39. 
14.

Nikolova-Damyanova, B., \& Handjieva, N. Quantitative determination of swertiamarin and gentiopicroside in Centaurium erythrea and C. turcicum by densitometry. Phytochemical Analysis 1996;7:14042.

15. Sharma Manish K et al., Significance of Plant Bitters In The Field of Pharmacognosy. Asian Journal of Pharmaceutical Technology \& Innovation, 01 (03); 2013; 01-14

16. European Pharmacopoeia. 9th ed. Strasbourg: European Directorate for the Quality of Medicines \& Health Care, 2017.

17. Alam P, Ali M, Singh R, Shakeel F. Estimation of swertiamarin in Enicostemma littorale and marketed formulations using HPLC-UV Method. Journal of Bioanalysis and Biomedicine. 2009;1(1):22-7. 18. Kshirsagar PR, Pai SR, Nimbalkar MS, Gaikwad NB. RP-HPLC analysis of seco-iridoid glycoside swertiamarin from different Swertia species. Natural product research. 2016 Apr 2;30(7):865-8. 19. ICH harmonised tripartite guideline Q2(R1). Validation of analytical procedures: text and methodology Q2(R1)," in Proceedings of the International Conference on Harmonization of Technical Requirements for Registration of Pharmaceuticals for Human Use, Geneva, Switzerland, 2005.

State Pharmacopoeia of Ukraine: in 3 volumes. Kharkiv: State enterprise "Ukrainian scientific pharmacopoeial center of medicines quality", 2-nd ed., Vol. 3., 2014

21. S. Gubar, A. Materiienko, V. Georgiyants, Olha Vasylieva, Ludas Ivanauskas Comparative analysis of the qualitative composition of secoiridoid glycosides in Centaurium erythraea Rafn. and Centaurium pulchellum (Sw) Druce herbs. The 9th International Conference on Pharmaceutical Sciences and Pharmacy Practice, 2018; 66.

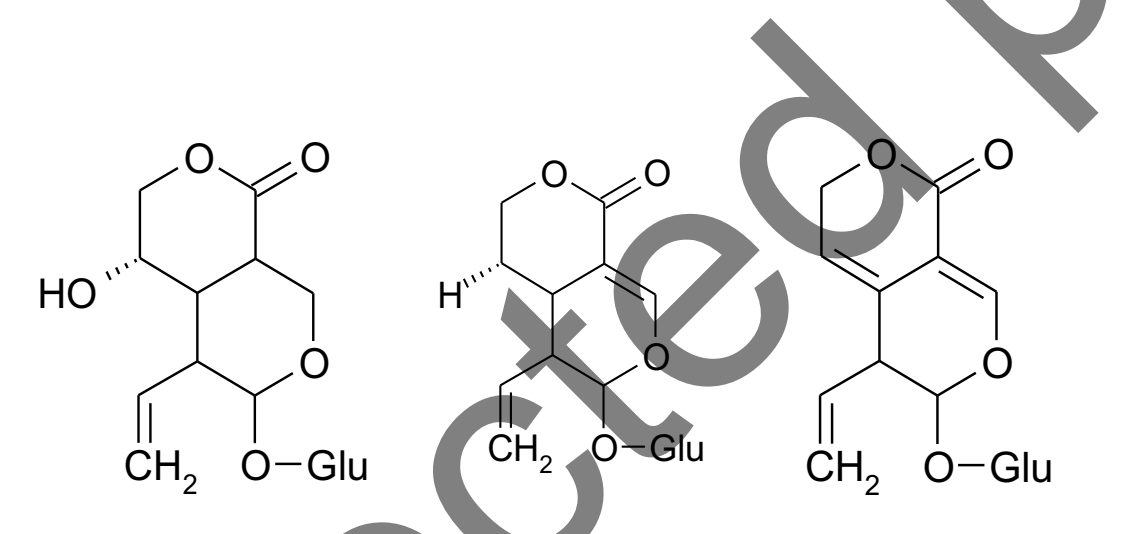

Swertiamarin Sweroside Gentiopicroside

Fig. 1. Structural formulas of secoiridoid glycosides of the Centaury 


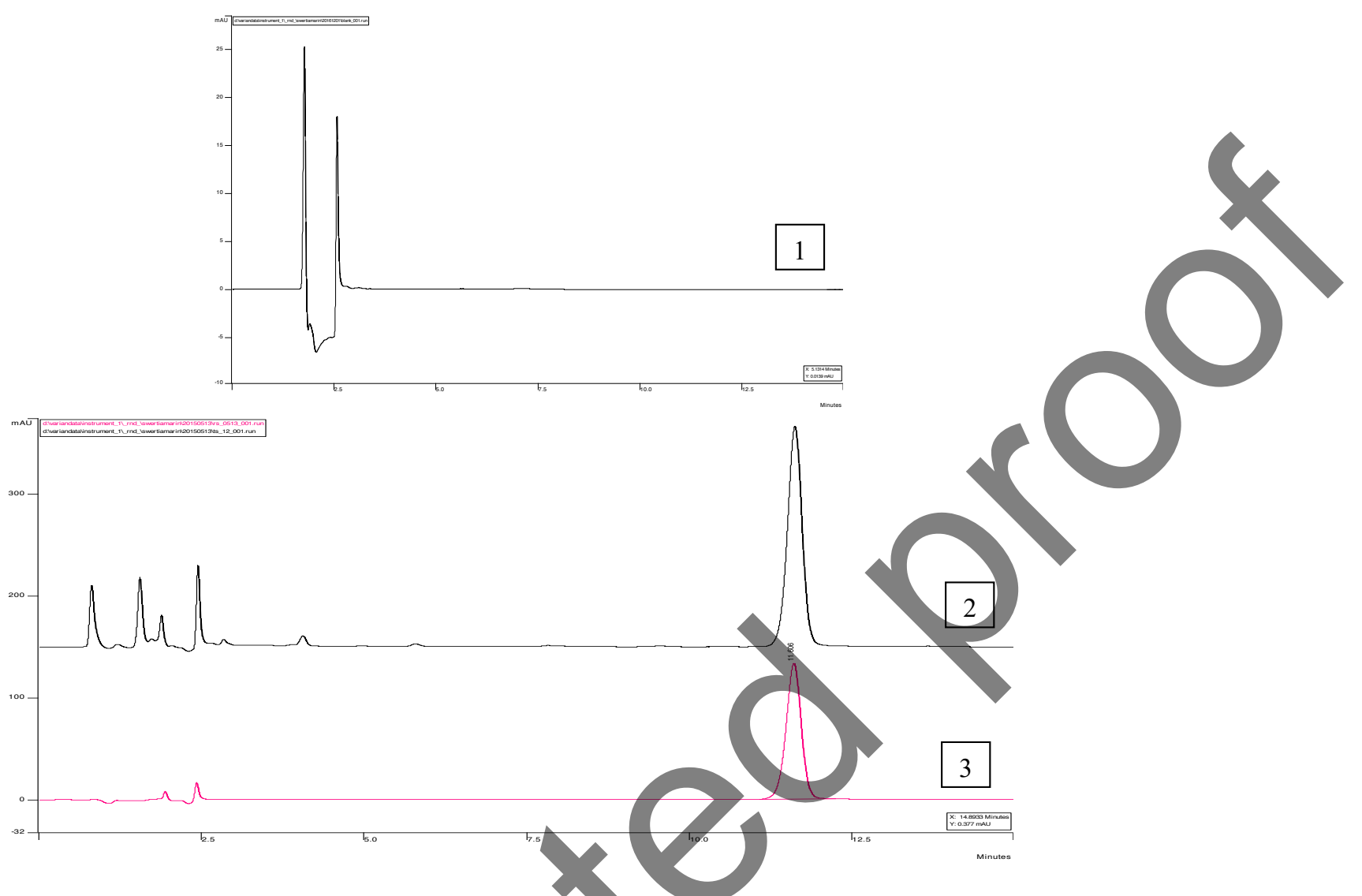

Fig. 2. Chromatograms of the blank solution (1), the test solution (2) and reference solution (3) 


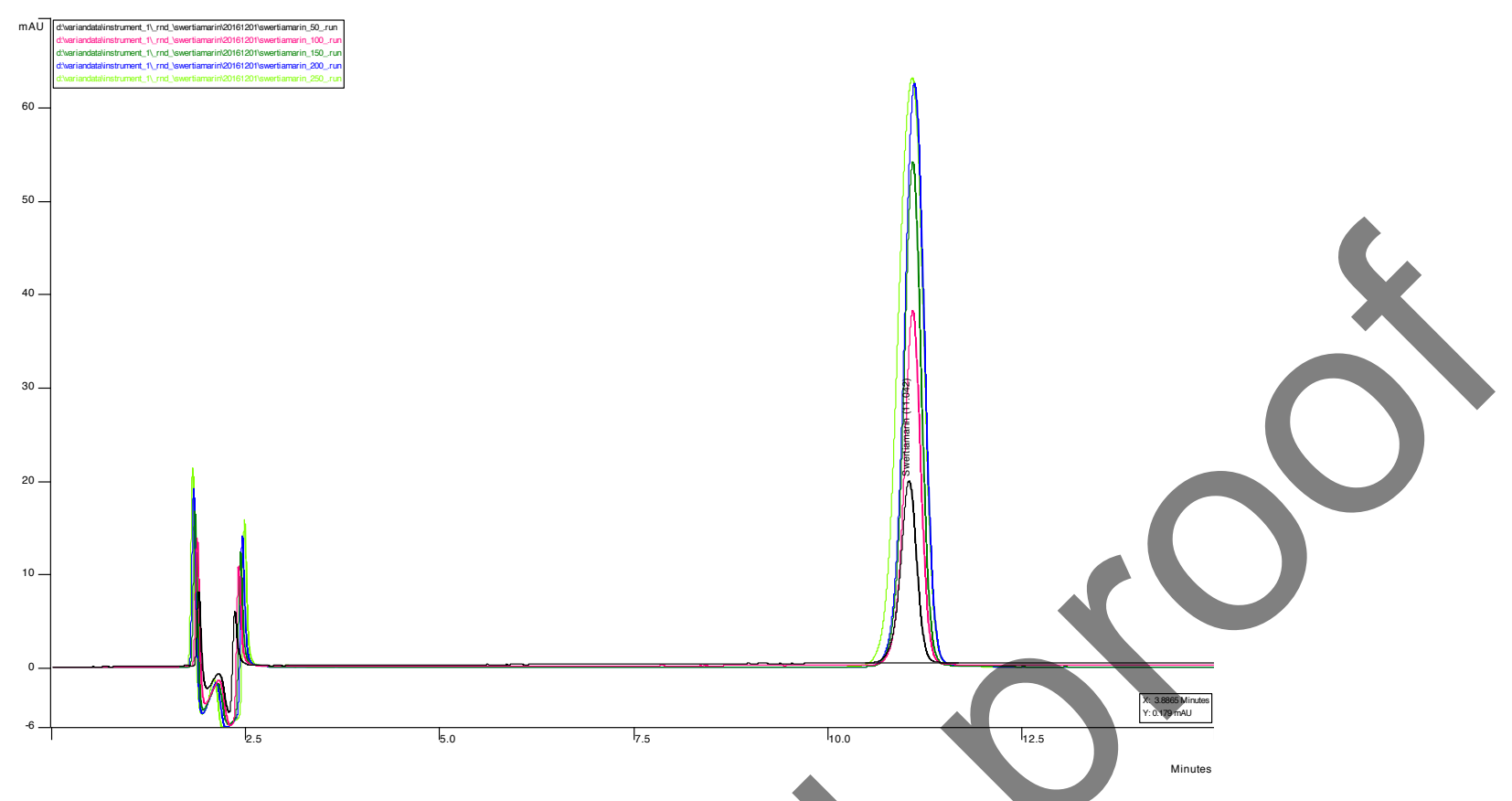

Fig. 3. Chromatogram of Swertiamarin solutions for linearity determination in the concentration range from $0.01 \mathrm{mg} / \mathrm{ml}$ to $0.05 \mathrm{mg} / \mathrm{ml}$

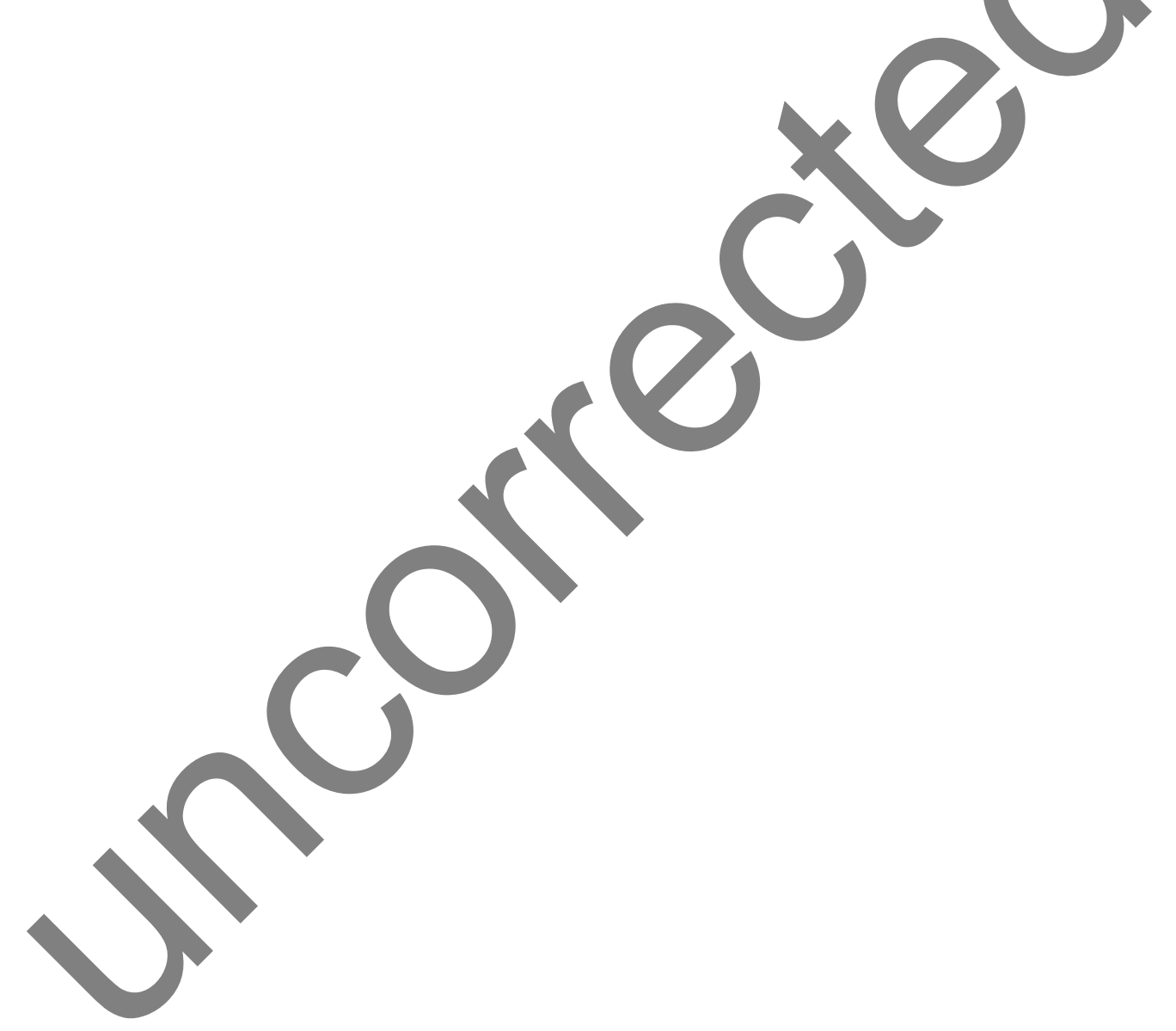




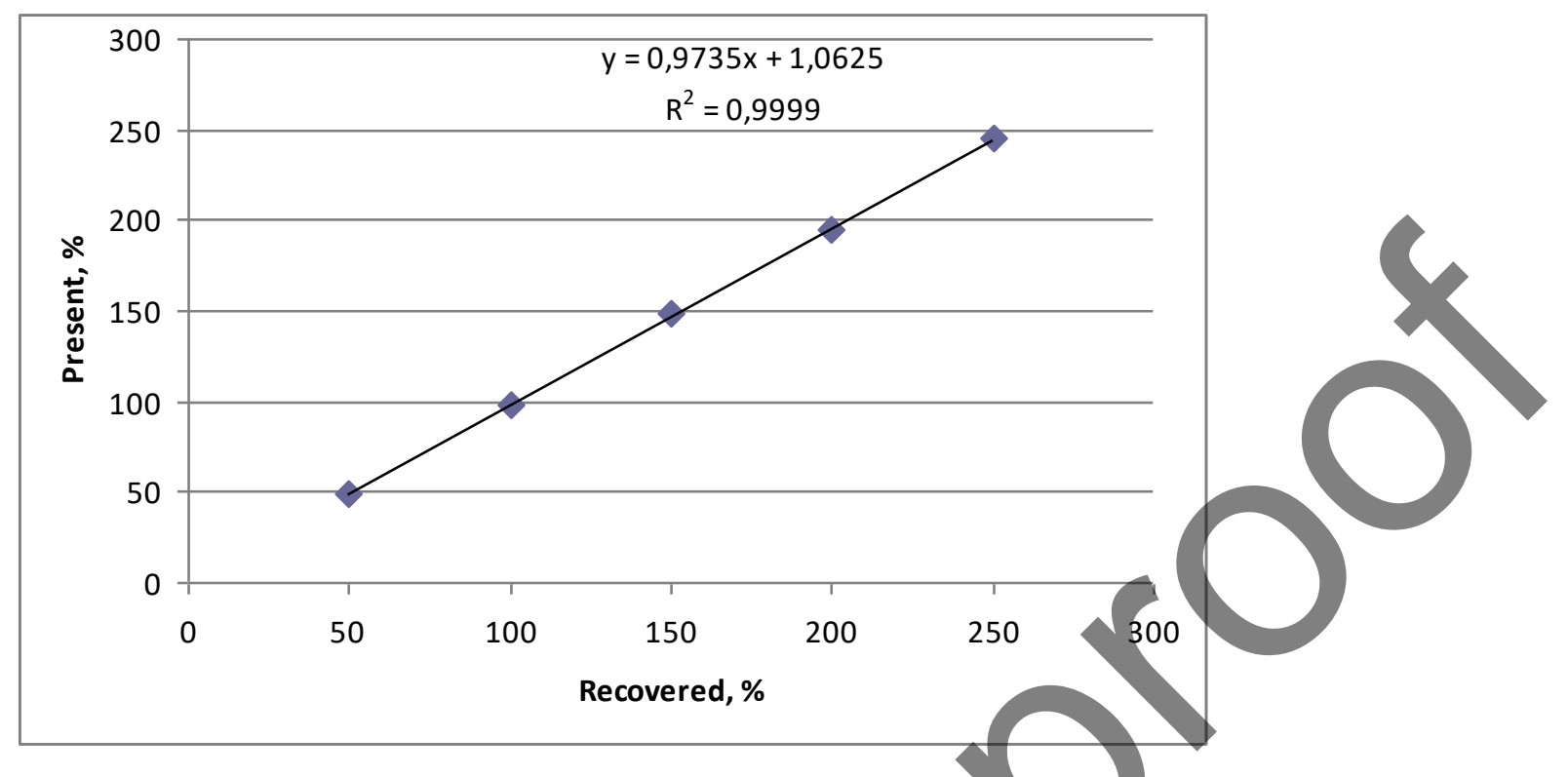

Fig. 4. The linearity curve of the Swertiamarin concentration by HPLC method 